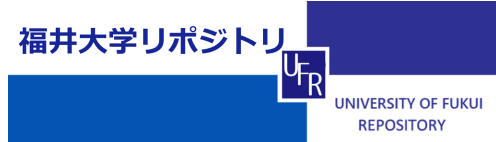

Vi sual i zati on of ul trasoni c wave fronts usi ng hol ogr aphi c i nt erfer onet ry

\begin{tabular}{|l|l|}
\hline 著者 & $\begin{array}{l}\text { OSH DA Yoshi hi r o, I WATA Koi chi , NAGATA Ryo, } \\
\text { UEDA Nasahi r o }\end{array}$ \\
\hline $\begin{array}{l}\text { j our nal or } \\
\text { publ i cat i on ti t l e }\end{array}$ & Appl i ed Opt i cs \\
\hline vol une & 19 \\
\hline nunber & 2 \\
\hline page r ange & $222-227$ \\
\hline year & $1980-01$ \\
\hline URL & ht t p: //hdl . handl e. net /10098/1445 \\
\hline
\end{tabular}




\title{
Visualization of ultrasonic wave fronts using holographic interferometry
}

\author{
Yoshihiro Oshida, Koichi Iwata, Ryo Nagata, and Masahiro Ueda
}

\begin{abstract}
Holographic interferometry with a phase-modulated reference beam is used to visualize a progressive uitrasonic wave in the interior of a transparent medium. The medium is illuminated with sheetlike light, and the light scattered from the plane of the light sheet is recorded in the hologram. The ultrasonic field on the plane is obtained from the reconstructed image, which provides the equiphase positions of the wave, i.e., wave fronts. Experiments were performed at $200-\mathrm{kHz}$ frequency. The effect of flow in the medium is estimated. An analysis of the optical system suggests that this method is applicable to ultrasonic waves with frequencies up to $\sim 6 \mathrm{MHz}$.
\end{abstract}

\section{Introduction}

Phase and amplitude of a small vibration on a rough surface can be detected by a technique in holographic interferometry with a sinusoidal phase-modulated reference beam. ${ }^{1-3}$ A progressive ultrasonic wave is a kind of vibration that usually has slow spatial variation of amplitude but rapid and continuous variation of phase. Thus, applying the above method for the progressive ultrasonic wave, we can expect to have reconstructed fringes indicating equiphase positions of the wave. ${ }^{4}$ The pattern obtained in this case shows the wave fronts of the wave.

To apply this method to the ultrasonic wave propagated in the interior of the medium, a substitute for the rough surface is necessary. For this purpose we can use the method adopted for measuring the flow velocity in a liquid ${ }^{5}$ : fine particles are suspended in the medium, and they are illuminated by sheetlike light obtained with cylindrical lenses. The plane illuminated by the sheetlike light plays the role of the rough surface.

To visualize the wave fronts of an ultrasonic wave, the schlieren technique can be used. ${ }^{6}$ The pattern obtained by this method, however, is the result of addition of information gained along the light propagation path.

M. Ueda is with Fukui University, Techniques Department, Education Faculty, Fukui, Japan; the other authors are with University of Osaka Prefecture, Department of Mechanical Engineering, College of Engineering, Sakai, Osaka, 591 Japan.
The schlieren technique, therefore, can only be applied to a 2-D wave field in which both amplitude and phase are constant along the light path. In contrast, by using the present holographic method with sheetlike light, we can obtain the information on the plane illuminated with sheetlike light. Three-dimensional information can be obtained by varying the position of the light sheet.

This method has been proposed, and a primary experimental result has been reported. ${ }^{4}$ The result, however, was not satisfactory in that a light beam was used instead of a light sheet. In this paper we shall explain the method in some detail and demonstrate those experimental results that visualize the progressive ultrasonic wave using the light sheet. We shall also give some analyses of the effect of flow in the medium and the frequency range of applicable ultrasound.

\section{Theory}

Consider the vibration in the form of a progressive wave as illustrated in Fig. 1. Figure 1(a) shows the spatial forms of the wave at times $t_{0}$ and $t_{0}+\Delta t$. The solid line means the wave position at time $t_{0}$, and this wave travels to the position of the broken line for time interval $\Delta t$. Figures $1(\mathrm{~b}), 1(\mathrm{c})$, and 1(d) show the temporal variations of the vibrations at three positions $P_{1}, P_{2}$, and $P_{3}$ in Fig. 1(a), respectively. As seen from (b)-(d), the initial phase of the vibration at each position varies continuously in the direction of the wave propagation. This continuous variation of phase is a difference of a progressive wave from a stationary wave whose phase changes discretely by $\pi$ in space. In a number of papers ${ }^{1,2}$ the sinusoidal phase-modulated reference beam technique was applied to phase detec- 
tion of the stationary wave. However, phase detection of the progressive wave has been demonstrated in only a few papers. 3,4

Figure 2 gives a schematic description of this method. Consider the visualization of the progressive ultrasonic

(a)

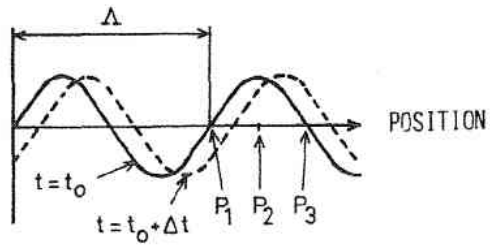

(b)

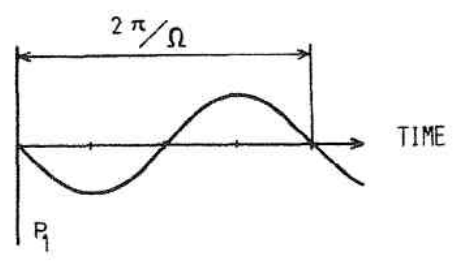

(c)

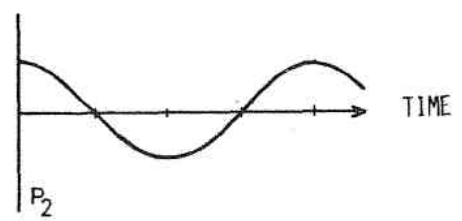

(d)

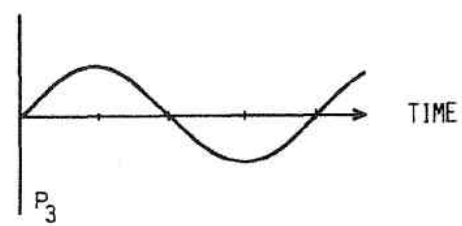

Fig. 1. Schematic illustration of the progressive wave: (a) spatial variation; (b) temporal variation at $P_{1}$; (c) temporal variation at $P_{2}$; (d) temporal variation at $P_{3}$.

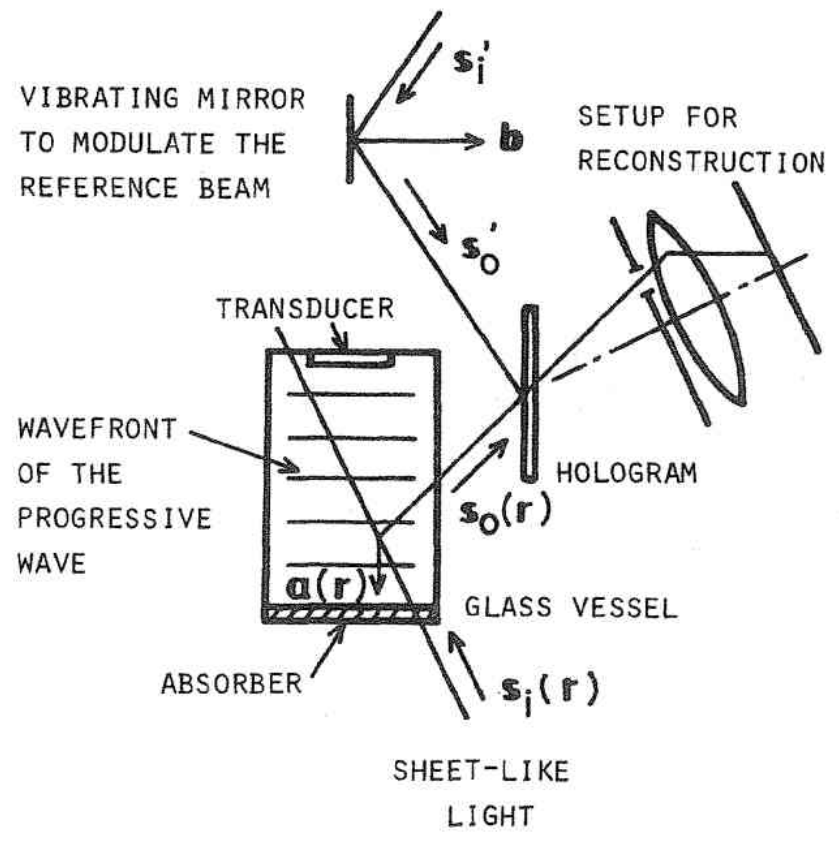

Fig. 2. Schematic description for phase-modulating holographic interferometry. wave radiated in the interior of the transparent medium by the transducer. The hologram is recorded with the light scattered on the light sheet and the phase-modulated reference beam. In Fig. $2, \mathbf{s}_{i}(\mathbf{r})$ is a unit vector in the propagation direction of the sheetlike light, and $\mathbf{s}_{\theta}(\mathbf{r})$ is a unit vector in the observation direction; $\mathbf{s}_{i}^{\prime}$ and $\mathbf{s}_{\text {, }}$ are unit vectors in the propagation direction of the light illuminating the vibrating mirror to modulate the reference beam and in the observation direction, respectively.

Assume that a point at position vector $\mathbf{r}$ on the light sheet is vibrating sinusoidally at angular frequency $\Omega$. Then the displacement of this point at time $t, \mathbf{d}(\mathbf{r}, t)$, can be expressed as

$$
\mathbf{d}(\mathbf{r}, t)=\mathbf{a}(\mathbf{r}) \cos [\Omega t+\mu(\mathbf{r})],
$$

where $\mathbf{a}(\mathbf{r})$ is the ultrasonic vibration amplitude, and $\mu(\mathbf{r})$ is the initial phase of the ultrasonic vibration. Amplitude a(r) varies slowly in space compared to phase $\mu(\mathbf{r})$. Similarly, the vibration of the mirror, which sinusoidally modulates the phase of the reference beam at the same angular frequency as that of the ultrasonic wave, is expressed as

$$
\mathbf{d}^{\prime}(t)=\mathbf{b} \cos (\Omega t+\phi),
$$

where $\mathbf{b}$ is the vibration amplitude, and $\phi$ is the vibration phase of the mirror.

In such circumstances, the irradiance $I(\mathbf{r})$ of the reconstructed image of point $\mathbf{r}$ can be written as $^{2}$

$$
I(\mathbf{r})=I_{s t}(\mathbf{r})=J_{0}^{2}\left[\frac{2 \pi}{\lambda} c(\mathbf{r})\right],
$$

where

$$
\begin{aligned}
c^{2}(\mathbf{r}) & =p^{2}(\mathbf{r})+q^{2}-2 p(\mathbf{r}) q \cos [\mu(\mathbf{r})-\phi] \\
p(\mathbf{r}) & =\left[\mathbf{s}_{o}(\mathbf{r})-\mathbf{s}_{i}(\mathbf{r})\right] \cdot \mathbf{a}(\mathbf{r}), \\
q & =\left(\mathbf{s}_{o}^{\prime}-\mathbf{s}_{i}^{\prime}\right) \cdot \mathbf{b}
\end{aligned}
$$

$I_{s t}(\mathbf{r})$ is the reconstructed irradiance obtained without the ultrasonic wave and the reference beam modulation, $J_{0}$ is the zero-order Bessel function of the first kind, and $\lambda$ is the wavelength of light. The quantities concerned with the reference beam modulation, i.e., the amplitude $q$ and the phase $\phi$, are constant in space.

The working point determined by the modulation amplitude of the reference beam is illustrated in Fig. 3.

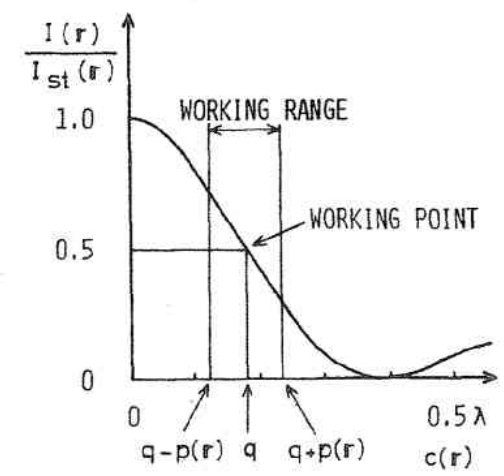

Fig. 3. Working point by reference phase modulation. 


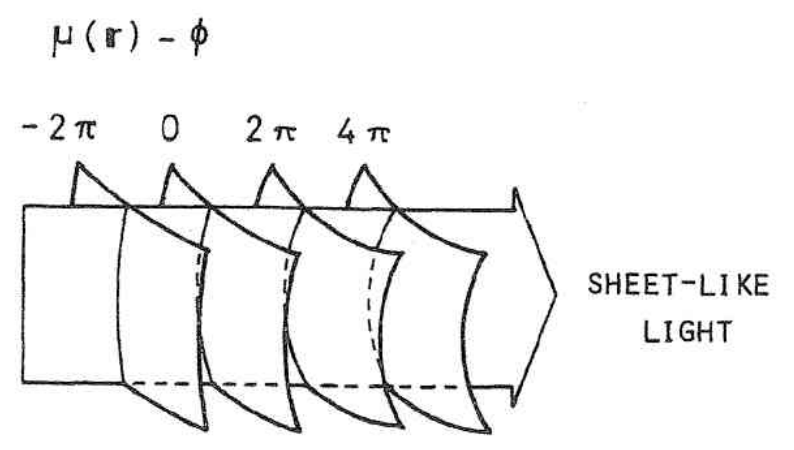

WAVEFRONTS OF THE

ULTRASONIC WAVE

Fig. 4. Intersections of the ultrasonic wave fronts with the plane of the light sheet.

For an ordinary ultrasonic wave the vibration amplitude is much smaller than the wavelength of light and the modulation amplitude, i.e., $p(\mathbf{r}) \ll \lambda$ and $p(\mathbf{r}) \ll q$. In such circumstances we may expand $I(\mathbf{r})$ as a power series in $p(\mathbf{r}) / \lambda$. Neglecting the quadratic and higherorder terms in $p(\mathbf{r}) / \lambda$, we obtain

$$
\begin{aligned}
I(\mathbf{r})= & I_{s t}(\mathbf{r})\left\{J_{0}^{2}\left(\frac{2 \pi}{\lambda} q\right)-\frac{4 \pi p(\mathbf{r})}{\lambda} \cos [\mu(\mathbf{r})-\phi]\right. \\
& \left.\times J_{\mathrm{o}}\left(\frac{2 \pi}{\lambda} q\right) J_{1}\left(\frac{2 \pi}{\lambda} q\right)\right\},
\end{aligned}
$$

where $J_{1}$ is the first-order Bessel function of the first kind. To measure small vibrations, the working point is normally selected at the steepest region of $J_{0}^{2}$, where

$$
J_{0}^{2}\left(\frac{2 \pi}{\lambda} q\right)=\frac{1}{2}, \quad J_{1}\left(\frac{2 \pi}{\lambda} q\right)=0.477 .
$$

Then Eq. (3) becomes

$$
I(\mathbf{r})=\frac{1}{2} I_{s t}(\mathbf{r})\left\{1-\frac{8.47}{\lambda} p(\mathbf{r}) \cos [\mu(\mathbf{r})-\phi]\right\} .
$$

Next, consider the meaning of the irradiance distribution of the reconstructed image. For an ultrasonic wave the vibration amplitude varies slowly in space compared with the phase. Therefore, the reconstructed irradiance depends on the vibration phase alone. Since the vibration phase of the ultrasonic wave varies by $2 \pi$ every wavelength, the same state of the vibration repeats at intervals of the wavelength. Hence, reconstructed irradiance $I(\mathbf{r})$ has the same value at those places whose intervals are the wavelength. These places, having the same intensity, correspond to the wave fronts. Since the illumination exists only on the light sheet, we can observe the reconstructed fringes at the intersections of the wave fronts with the plane of the light sheet as illustrated in Fig. 4. Altering the position of the plane illuminated by the light sheet, we can obtain a 3-D ultrasonic field.

\section{Experimental Setup and Results}

The optical arrangement used for the experiments is shown in Fig. 5. An ultrasonic wave field is formed in the glass vessel by transducer $T d_{1}$. The sheetlike light formed through cylindrical lenses $L_{2}$ and $L_{3}$ illuminates the medium in the glass vessel. The vibration frequency is $200 \mathrm{kHz}$. The other transducer $T d_{2}$, which modulates the reference beam, is driven by the same oscillator as transducer $T d_{1}$.

Mirror $M_{2}$, beam splitter $B S_{2}$, and transducer $T d_{2}$ constitute the Twyman-Green interferometer. This interferometer is used for measuring the vibration amplitude of transducer $T d_{2}$. Visibility $V$ of the fringes is related to the vibration amplitude of $T d_{2}$ through the zero-order Bessel function of the first kind, that is,

$$
V=\left|J_{0}\left(\frac{2 \pi}{\lambda} q\right)\right| \text {. }
$$

The vibration amplitude is adjusted so that $q \simeq \lambda / 5$, corresponding to

$$
J_{0}^{2}\left(\frac{2 \pi}{\lambda} q\right) \simeq \frac{1}{2} .
$$

The first example is the visualization of the ultrasonic wave propagated in water. The glass vessel is filled with water-suspended fine alumina particles. The Ar laser has a $\lambda=514.5 \mathrm{~nm}$.

If the ultrasonic wave is propagated in the liquid, flow is caused by the radiation pressure. To eliminate the influence of this flow, exposure time must be short, as discussed below. However, to expose the photographic plate sufficiently the time must be longer than a certain value: $1 / 250 \mathrm{sec}$ in our experiment. Therefore, the exposure time was divided into two parts of $1 / 500 \mathrm{sec}$ each.

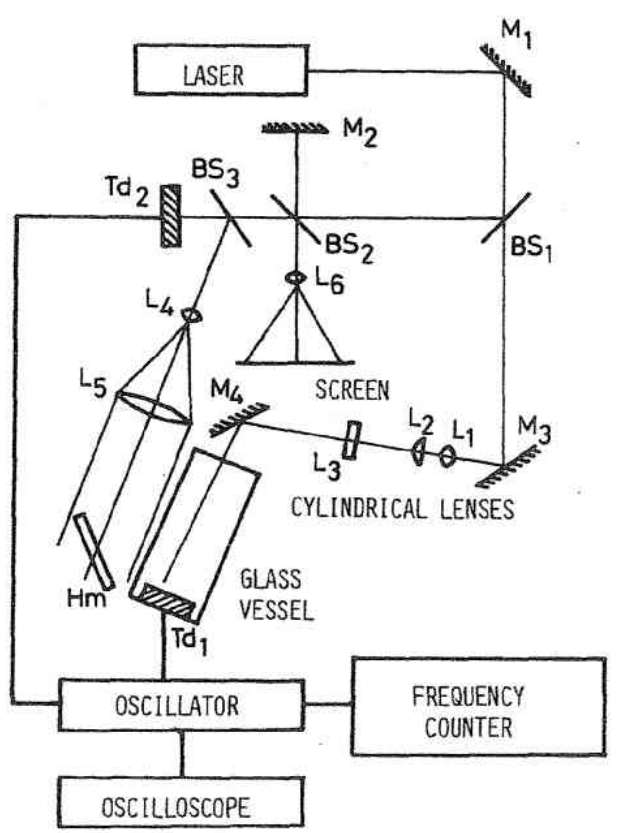

Fig. 5. Experimental setup: $L$, lens; $M$, mirror; $B S$, beam splitter; $H \mathrm{~m}$, hologram; $\mathrm{Td}$, transducer. 
The reconstructed image is shown in Fig. 6. The ultrasonic wave is traveling from right to left. The broad bright and dark fringes indicate the wave fronts. The interval of the fringes is $\sim 7 \mathrm{~mm}$, which agrees with the wavelength calculated with $1448-\mathrm{m} / \mathrm{sec}$ propagation velocity (at $10^{\circ} \mathrm{C}$ ) and $200-\mathrm{kHz}$ frequency.

The next example is the wave in gelatin in the gel state. In this case no flow is caused by the ultrasonic wave, so that exposure time may be long. The hologram plate was exposed for $60 \mathrm{sec}$ with light from a $\mathrm{He}-\mathrm{Ne}$ laser with $\lambda=632.8 \mathrm{~nm}$.

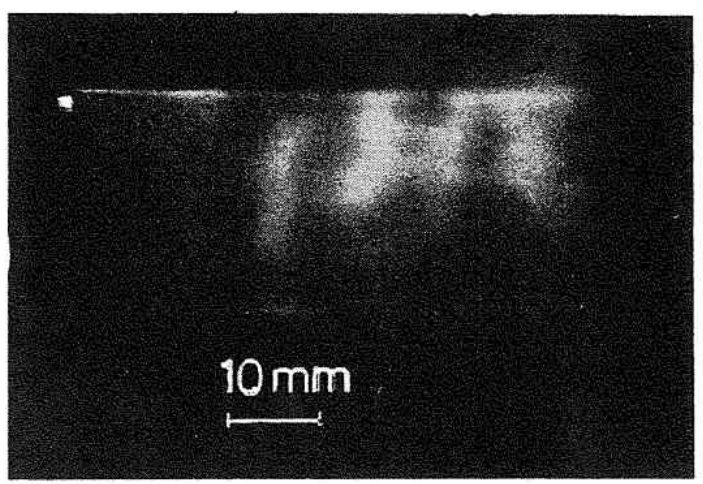

Fig. 6. Reconstructed image in water.

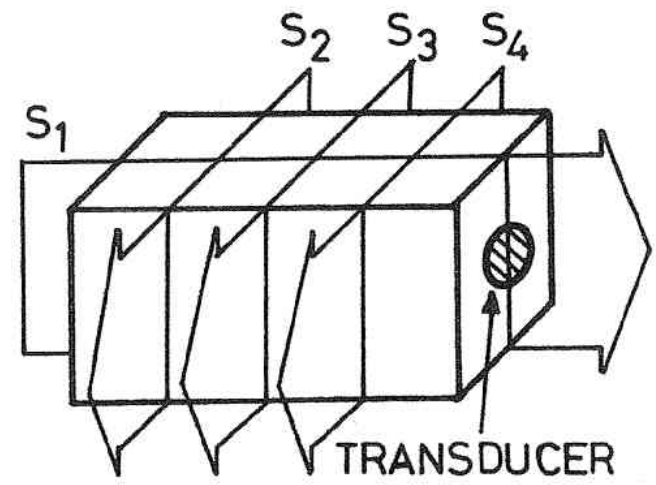

(a)

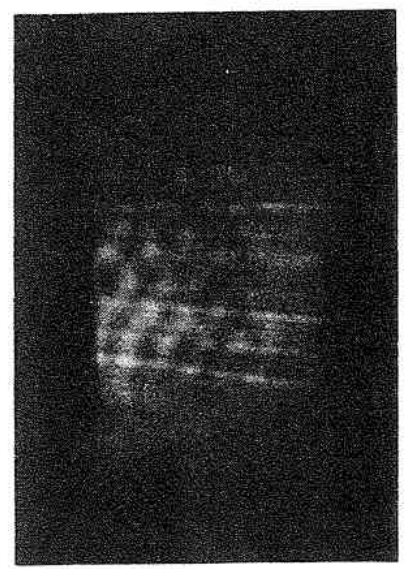

(c)
The reconstructed images are shown in Fig. 7. The illuminating directions of the light sheet are illustrated in Fig. 7(a). Figures 7(b), 7(c), 7(d), and 7(e) are the reconstructed images at four sections $S_{1}, S_{2}, S_{3}$, and $S_{4}$, respectively. The ultrasonic wave is traveling from right to left in Fig. 7(b), and it is traveling perpendicular to the figure plane in Figs. 7(c), 7(d), and 7(e).

As seen from Fig. 7(b), the ultrasonic wave radiated from the transducer is propagated in the form of a spherical wave, and the wave is reflected at the upper surface of the medium and the bottom of the vessel. Figure 7(e) shows the ultrasonic field of the cross section in front of the transducer. Since a spherical wave is radiated, the resultant fringes are concentric circles. Figures 7(c) and 7(d) are the cross sections farthest from the transducer. The reconstructed images consist of checkered patterns, resulting from interference of the waves propagated directly and reflected at the upper surface, bottom, and both side walls.

\section{Discussion}

\section{A. Influence of the Flow}

For an ultrasonic wave propagated in liquid, flow is caused by radiation pressure. It disturbs the reconstructed image. We shall explain the influence of flow quantitatively in this section.
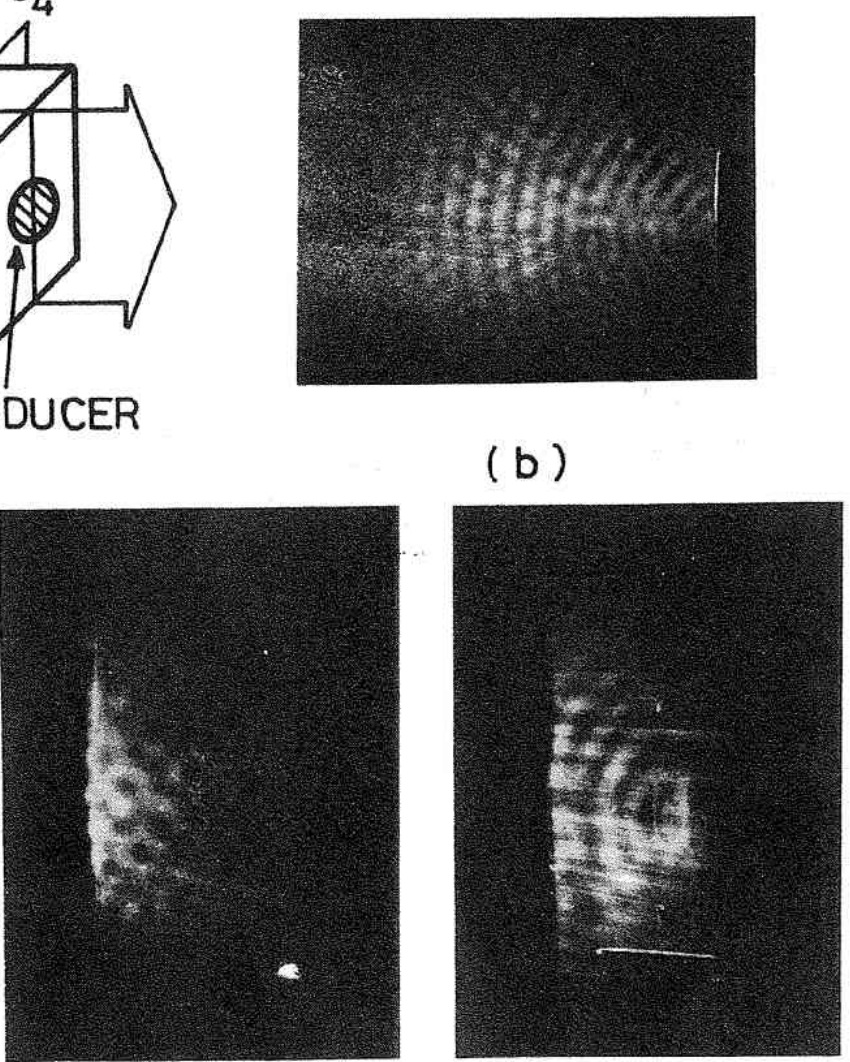

(d)

\section{(e)}

Fig. 7. Reconstructed images in gelatin: (a) illuminating directions of the light sheet; (b) image on $S_{1}$; (c) image on $S_{2}$; (d) image on $S_{3}$; (e) image on $S_{4}$. 
Consider that the object at position $\mathbf{r}$ moves straight at velocity $\mathbf{v}(\mathbf{r})$ and in addition vibrates sinusoidally as expressed in Eq. (1). Then displacement $\mathbf{d}_{v}(\mathbf{r}, t)$ at time $t$ and initial position $\mathbf{r}$ is written as

$$
\mathbf{d}_{v}(\mathbf{r}, t)=\mathbf{a}(\mathbf{r}) \cos [\Omega t+\mu(\mathbf{r})]+\mathbf{v}(\mathbf{r}) t .
$$

The reconstructed irradiance $I(\mathbf{r})$ obtained by time-averaged holography with the object beam phase-modulated by Eq. (6) and the reference beam modulated by Eq. (2) is expressed as

$I(\mathbf{r})=I_{s t}(\mathbf{r}) \frac{1}{T} \int_{-\frac{T}{2}}^{\frac{T}{2}} \exp i \frac{2 \pi}{\lambda}\left\{c(\mathbf{r}) \cos [\Omega t+\psi(\mathbf{r})]+v^{\prime}(\mathbf{r}) t\right\} d t$,

where

$$
\left.\begin{array}{l}
\left.\psi(\mathbf{r})=\mu(\mathbf{r})-\sin ^{-1}\left\{\frac{q}{c(\mathbf{r})} \sin [\mu(\mathbf{r})-\phi]\right\}\right\}, \\
v^{\prime}(\mathbf{r})=\left[\mathbf{s}_{o}(\mathbf{r})-\mathbf{s}_{i}(\mathbf{r})\right] \cdot \mathbf{v}(\mathbf{r})
\end{array}\right\}
$$

and $T$ is the exposure time. If there are many cycles of ultrasonic vibration during exposure time and the displacement due to the velocity $\mathbf{v}(\mathbf{r})$ during the vibrational period is much smaller than the wavelength of light, i.e.,

$$
T \gg \frac{2 \pi}{\Omega}, \quad \frac{2 \pi v^{\prime}(\mathbf{r})}{\Omega} \ll \lambda,
$$

Eq. (7) can be calculated as

$$
I(\mathbf{r})=I_{s t}(\mathbf{r}) J_{0}^{2}\left[\frac{2 \pi}{\lambda} c(\mathbf{r})\right]\left[\frac{\sin u(\mathbf{r})}{u(\mathbf{r})}\right]^{2},
$$

where

$$
u(\mathbf{r})=\frac{\pi}{\lambda} v^{\prime}(\mathbf{r}) T
$$

Equation (10) indicates that the reconstructed image consists of the superposition of two kinds of fringes, one of which gives information about ultrasonic vibration and the other about transfer velocity. The former is a term of the Bessel function, and the latter is a term of the sinc function. Since the fringes caused by flow disturb the visualization of the ultrasonic field, $u(\mathbf{r})$ must be small enough that the term of the velocity component in Eq. (10) can be regarded as unity.

For example, if we consider that

$$
\left[\frac{\sin u(\mathbf{r})}{u(\mathbf{r})}\right]^{2}
$$

must be larger than 0.8 ,

$$
\frac{\pi}{\lambda} v^{\prime}(\mathbf{r}) T<0.74
$$

If $v^{\prime}(\mathbf{r})=60 \mu \mathrm{m} / \mathrm{sec}$, the exposure time must be shorter than $1 / 500$ sec. On the other hand, from Eqs. (9) and (12) the relation

$$
\frac{2 \pi^{2}}{\lambda \Omega} v^{\prime}(\mathbf{r}) \ll 0.74
$$

must be satisfied. Therefore, the permissible flow velocity is limited by the frequency of the ultrasonic wave. If $\Omega /(2 \pi)=200 \mathrm{kHz}, v^{\prime}(\mathbf{r})$ must be much smaller than $20 \mathrm{~mm} / \mathrm{sec}$.

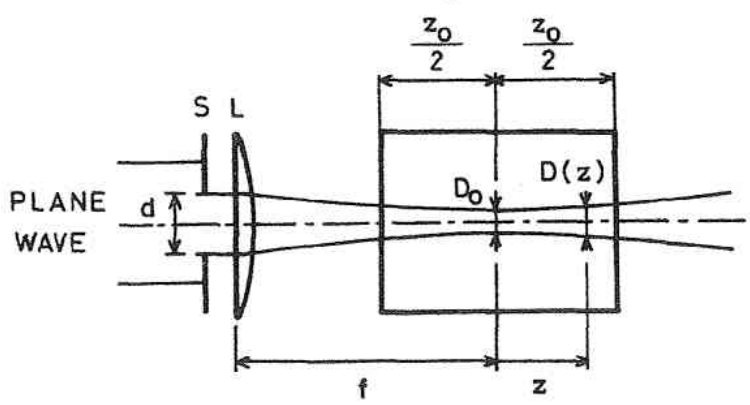

Fig. 8. Schematic diagram of top view of sheetlike light: $S$, slit of width $d ; L$, cylindrical lens of focal length $f$.

\section{B. Limitation of the Frequency of the Observable Ultrasonic Wave}

To detect the vibrational phase of the ultrasonic wave, the thickness of the light sheet should be much less than that of the ultrasonic wavelength. Hence, the detectable frequency range is limited by the thinnest possible sheetlike light.

Consider the arrangement illustrated in Fig. 8, which shows a cross section of the sheetlike light extending perpendicular to the plane of the figure. The light is excluded by slit $S$ of width $d$ placed against a cylindrical lens of focal length $f$. Measure the $z$ coordinate toward the right from the focal plane of the lens $L$. The measuring region occupies from $z=-z_{0} / 2$ to $z=z_{0} / 2 ; D(z)$ indicates the thickness of the light sheet at position $z$.

Thickness $D_{0}$ at $z=0$ is written, using the relation for Fraunhofer diffraction,

$$
D_{0}=2 f \lambda / d \text {. }
$$

The thickness at position $z$ far from the focal plane is expressed by the boundary thickness of the geometrical shadow, i.e.,

$$
D(z)=(d / f)|z|,
$$

but

$$
D(z) \geq D_{0} .
$$

$D(z)$ has the maximum value at positions $z= \pm z_{0} / 2$.

To detect the phase of the wave, the ultrasonic wavelength $\Lambda$ must be longer than the maximum light-sheet thickness:

$$
\Lambda=\alpha D\left( \pm z_{0} / 2\right),
$$

where $\alpha$ is a constant value much larger than unity. The maximum thickness is given by Eq. (17) for a certain wavelength $\Lambda$. When $D\left( \pm z_{0} / 2\right)=D_{0}$, measuring region $z_{0}$ has a maximum value for a given maximum thickness. Then from Eqs. (14) and (17) $f / d$ satisfies the relation

$$
f / d=\Lambda /(2 \alpha \lambda) .
$$

Substituting Eqs. (17) and (18) into Eq. (15), we obtain the relation

$$
z_{0}=\Lambda^{2} /\left(\lambda \alpha^{2}\right) .
$$


Equation (19) provides the maximum possible value of the measuring region. The maximum number $N$ of fringes obtained within region $z_{0}$ is given by dividing $z_{0}$ by wavelength $\Lambda$;

$$
N=\left(1 / \alpha^{2}\right) /(\Lambda / \lambda) .
$$

Equation (20) indicates that the number of detectable fringes is limited by the ratio of the acoustical wavelength to the optical wavelength. Conversely, if the desirable number of fringes is given, the detectable wavelength of the wave is limited by Eq. (20). That wave whose wavelength is less than the value obtained from Eq. (20) is unmeasurable, i.e., $\Lambda$ obtained from Eq. (20) indicates the limitation of the wavelength of the observable ultrasonic wave.

For example, consider the limitation of the ultrasonic wave in water. The optical wavelength $\lambda$ of a $\mathrm{He}-\mathrm{Ne}$ laser is $475.8 \mathrm{~nm}$ in water. If $N=20$ and $\alpha=5$, the minimum value of the wavelength of an observable acoustic wave is $\Lambda=0.24 \mathrm{~mm}$. This wavelength corresponds to a frequency of $6 \mathrm{MHz}$.

\section{Conclusion}

A method of visualizing the ultrasonic field in the interior of a transparent medium is presented. The experimental results, which visualize ultrasonic waves propagated in water and gelatin, are demonstrated. A 3-D ultrasonic field can be visualized by this method. Analysis shows that the displacement caused by the flow in the test fluid during an ultrasonic vibration period must be much smaller than the wavelength of light. The applicable frequency of the ultrasonic wave is limited to $\sim 6 \mathrm{MHz}$ by the thinnest possible sheetlike light.

The authors thank Kimio Kubo of Sharp Corporation for helpful advice on the electronic circuits. This research was supported by a Grant-in-Aid for Scientific Research from the Ministry of Education of Japan.

\section{References}

1. C. C. Aleksoff, Appl. Phys. Lett. 14, 23 (1969).

2. D. B. Neumann, C. F. Jacobson, and G. M. Brown, Appl. Opt. 9, 1357 (1970).

3. A. F. Metherell, in Acoustical Holography, P. S. Green, Ed. (Plenum, New York, 1974), Vol. 5, pp. 41-58.

4. M. Ueda, S. Okuno, Y. Oshida, K. Iwata, and R. Nagata, Optik (Stuttgart) 52, 71 (1978-79).

5. K. Iwata, T. Hakoshima, and R. Nagata, J. Opt. Soc. Am. 67, 1117 (1977).

6. W. E. Moore and J. A. Bucaro, J. Acoust. Soc. Am. 63, 60 (1978). 